\title{
Extranodal NK/T-cell lymphoma, nasal type
}

\author{
Prescilla B. Wood, MD, Sachin R. Parikh, MD, and John R. Krause, MD
}

We report a case of extranodal NK/T-cell lymphoma, nasal type, which is rare in the United States and Europe. It is more prevalent in Asians and Native Americans of Mexico, Central America, and South America. A 30-year-old Southeast Asian man with facial swelling, fever, and unintentional weight loss was found to have leukopenia and thrombocytopenia. He underwent endoscopic sinus surgery, which confirmed extranodal NK/T-cell lymphoma, nasal type, and a blood and bone marrow examination, which was negative for involvement but yielded the diagnosis of alpha-E thalassemia. The patient received chemotherapy, radiotherapy, and a stem cell transplant with $100 \%$ engraftment.

A

30-year-old Southeast Asian male with no significant medical history presented to a primary care physician with a 2-month history of fever up to $102^{\circ} \mathrm{F}$, an unintentional 20-pound weight loss over 8 weeks, and left facial swelling around the left nasolabial fold. He was given oral antibiotics, which did not resolve the fever, and his facial swelling continued to worsen. He also saw a hematologist for leukopenia and thrombocytopenia, found on his initial presentation to the primary care physician, and was prescribed Neupogen. No bone marrow biopsy was obtained at that time.

Upon referral to an otolaryngologist, he was admitted to an outside hospital with moderate leukopenia (white blood cell count, $2.9 \mathrm{~K} / \mathrm{uL}$ ), slight anemia (hemoglobin, $13 \mathrm{~g} / \mathrm{dL}$ ), slight thrombocytopenia (platelet count, $102 \mathrm{~K} / \mathrm{uL}$ ), and mildly elevated transaminases (aspartate aminotransferase, $184 \mathrm{U} / \mathrm{L}$; alanine aminotransferase, $207 \mathrm{U} / \mathrm{L}$ ). The number of leukocytes increased after Neupogen was given (white blood cell count, $4.2 \mathrm{~K} / \mathrm{uL})$, but hemoglobin $(9.2 \mathrm{~g} / \mathrm{dL})$ and platelet counts $(51 \mathrm{~K} / \mathrm{uL})$ worsened. He was transferred to Baylor University Medical Center at Dallas. Despite taking intravenous antibiotics for several days, his left facial swelling increased.

Upon admission, the patient denied diplopia but reported decreased visual acuity in his left eye. He denied nausea or vomiting but had complaints of painful swallowing and mild headaches. He denied any bleeding, bruising, or enlarging lymph nodes. He was born in Thailand, was of Laotian descent, and had been living in the United States since he was 1 year old. On physical examination, he had left hemifacial edema involving the orbit, the cheek, and the parotid region, with severely decreased vision in the left eye. Preauricular lymphadenopathy, poor dentition with several broken and decayed teeth, and some superficial ulceration in the palate were noted. No cervical, supraclavicular, axillary, or inguinal lymphadenopathy was appreciated. The spleen was palpable at approximately $4 \mathrm{~cm}$ below the costal margin.

The patient's blood counts remained unchanged with a minimally elevated reticulocyte count $(2.1 \%)$. In light of the absolute lymphocytopenia $\left(416\right.$ cells $/ \mathrm{mm}^{3}$ ), the CD4 count was decreased $\left(171 \mathrm{cells} / \mathrm{mm}^{3}\right)$ but was of normal percentage (41\%). Acute hepatitis was ruled out; however, the chronic panel was reactive for hepatitis B core antibody and surface antibody with negative surface antigen, indicating immunization by prior infection. The Epstein-Barr virus (EBV) was detected at 1300 copies $/ \mathrm{mL}$.

A computed tomography (CT) scan of the soft tissues of the neck found pansinus inflammatory changes concerning for fungal sinusitis with invasive characteristics, particularly within the left paranasal sinuses with extension into the deep facial soft tissues and orbital apex (Figure 1). Severe dental, periodontal, and endodontal disease and an associated mild proptosis on the left side were noted. There was also a concerning narrowing of the airway at the level of the oropharynx, so the patient was taken urgently to surgery for diagnosis. Endoscopic sinus surgery was performed, in conjunction with the extraction of 12 teeth by the oral and maxillofacial surgery service.

With a nasopharyngeal mass and pansinus inflammation, the differential diagnosis based on clinical findings would include a fungal infection, nasopharyngeal carcinoma, or lymphoma. An abdominal ultrasound showed splenomegaly at $16.8 \mathrm{~cm}$, with a normal-sized liver. Given the patient's fevers, splenomegaly, and weight loss, lymphoma with sympathetic/ retrograde sinusitis was of concern.

Histologic examination of the specimens from the left sinuses found an atypical angioinvasive lymphoid infiltrate

From the Departments of Pathology (Wood, Krause) and Radiology (Parikh), Baylor University Medical Center at Dallas and Baylor Charles A. Sammons Cancer Center.

Corresponding author: Prescilla B. Wood, MD, Hematopathology Fellow, Department of Pathology, Baylor University Medical Center at Dallas, 3500 Gaston Avenue, Dallas, Texas 75246 (e-mail: PresciWo@BaylorHealth.edu). 

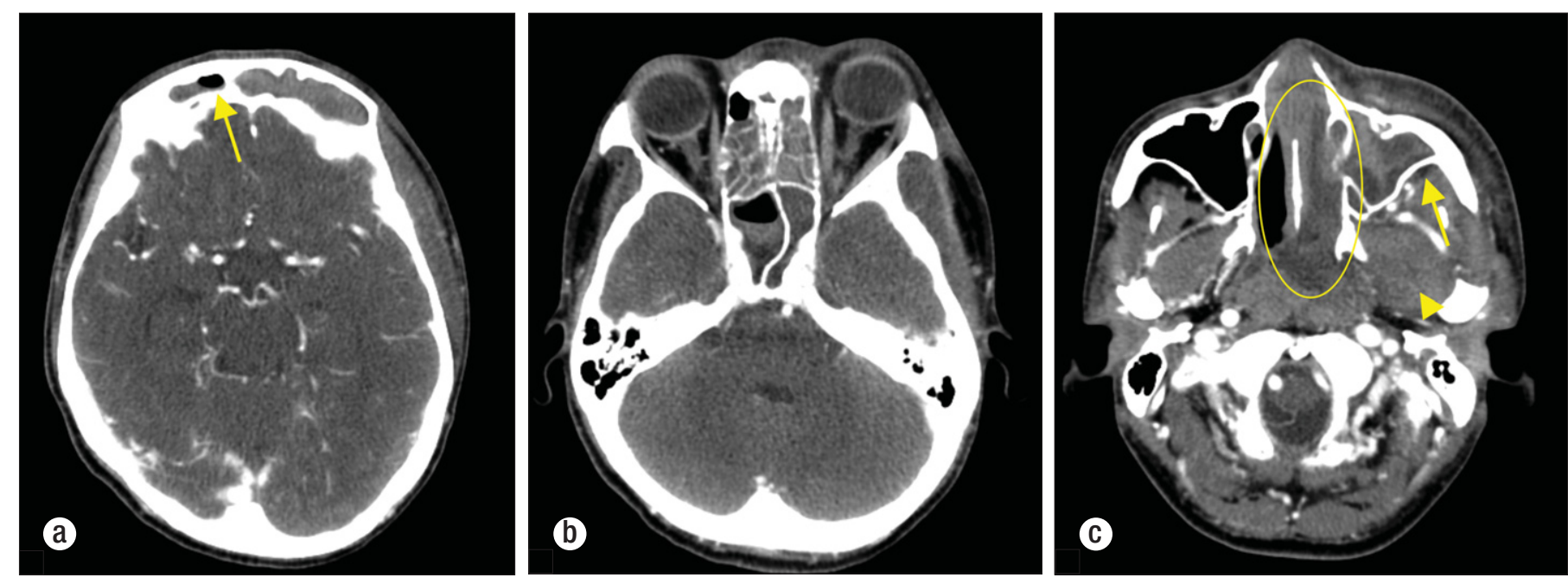

Figure 1. CT of the neck with contrast. (a) There is subtotal opacification of the frontal sinuses, with a small fluid level on the right (arrow). (b) The sphenoid and ethmoid sinuses are subtotally opacified with a fluid level in the right sphenoid sinus consistent with acute sinusitis. (c) The left maxillary sinus is completely opacified with increased intrinsic density centrally, which can be seen with fungal colonization or inspissated secretions. In addition, the opacities expand into the adjacent left nasal cavity via the osteomeatal unit (circle). There is asymmetric opacification of the left retroantral fat (arrow) and expansion of the left lateral pterygoid muscle (arrowhead) secondary to the patient's invasive fungal sinusitis.

comprising small- to medium-sized cells with irregular nuclei, inconspicuous nucleoli, and moderate pale to clear cytoplasm. The infiltrate was present in a background of severe chronic sinusitis with prominent areas of ulceration and necrosis. No fungal elements were identified on Gömöri methenamine silver stain, and no viral cytopathic effect was appreciated, although the in situ hybridization for EBV-encoded RNA (EBER) was strongly and diffusely positive. Upon further characterization, the atypical lymphoid infiltrate was positive for CD3, CD56, TIA-1, and granzyme-B, characteristic of NK cells or activated T lymphocytes, and was negative for CD57 (Figure 2). A diagnosis of extranodal NK/T-cell lymphoma, nasal type, was rendered. Molecular studies for B- and T-cell gene rearrangement on the left sinus contents were negative. With a lactic acid dehydrogenase level of $566 \mathrm{U} / \mathrm{L}$, the International Prognostic Index was low risk (1 point).

During his hospitalization, the patient had persistent pancytopenia. A staging bone marrow biopsy was performed. A peripheral blood smear showed a marked hypochromic microcytosis with occasional schistocytes, acanthocytes, and numerous elliptocytes. Polychromasia and fine basophilic stippling were present in the red cells, and occasional Döhle bodies were seen within the neutrophils. The bone marrow was appropriately cellular with no morphologic evidence of malignancy. Flow cytometry performed at Baylor University Medical Center at Dallas also found no evidence of a hematolymphoid malignancy. Cytogenetic analysis found a normal male karyotype $(46, \mathrm{XY})$.

In this patient with severe pancytopenia and extensive red cell changes, the differential diagnosis would include iron deficiency anemia, hemoglobinopathy, and thalassemia as possible etiologies. Iron stores were adequate, but the hemoglobin electrophoresis was reported as $77.9 \% \mathrm{~A} 1,4.4 \% \mathrm{~A} 2,0.0 \% \mathrm{~F}$, and $17.7 \%$ "other" hemoglobin, most likely hemoglobin E. The amount of hemoglobin E, too low for heterozygosity, in addition to the mean corpuscular volume (69 fL), suggested an accompanying thalassemia. With only A1, a small amount of $\mathrm{A} 2$, and $\mathrm{E}$ present, this patient most likely has an alpha-E thalassemia with two alpha chain deletions.

The patient was discharged home on hospital day 27. A CHOP chemotherapy regimen (cyclophosphamide, hydroxydaunorubicin [doxorubicin], Oncovin [vincristine], and prednisone/prednisolone) was started, with subsequent radiotherapy. Progressively, his swelling reduced and he improved. Due to left eye infiltration by the NK cell lymphoma, the patient rapidly developed complete vision loss. High-dose steroids were started but were unsuccessful in restoring any vision.

A positron emission tomography-CT scan performed 2 months after diagnosis showed a positive response to interval therapy with a significant decrease in intensity of hypermetabolic disease involving the left paranasal sinuses, nasal cavity, and oropharynx when compared with the pretreatment scan performed in August 2010. In January 2011, he received an allogeneic stem cell transplant and, on last examination, had $100 \%$ engraftment. The EBV titers performed in February and March 2011 showed nondetectable levels.

\section{DISCUSSION}

This case illustrates the need for a high index of suspicion in the diagnosis of NK-cell lymphomas. Extensive necrosis and hemorrhage are often superimposed on infection and are frequently dismissed as an infectious process. The location in the midline of the head provides a clue to lymphoma. Extranodal NK/T-cell lymphoma has an extranodal presentation, with the upper aerodigestive tract (nasal cavity, nasopharynx, paranasal sinuses, and palate) most commonly involved. The skin, soft tissue, gastrointestinal tract, and testis are the preferential extranasal sites of involvement. This entity has also been referred to as lethal midline granuloma, polymorphic reticulosis, angiocentric immunoproliferative lesion, and 

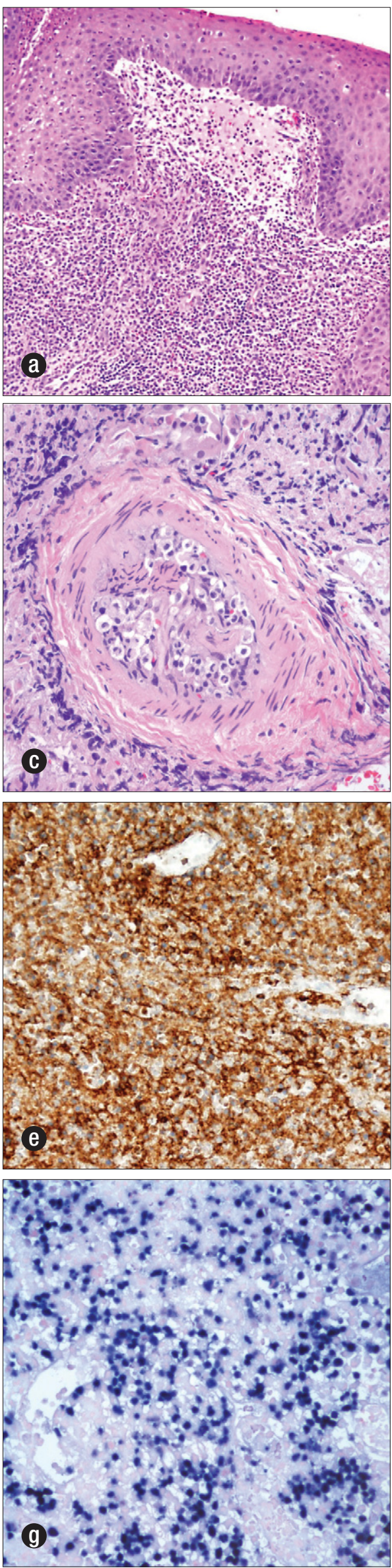
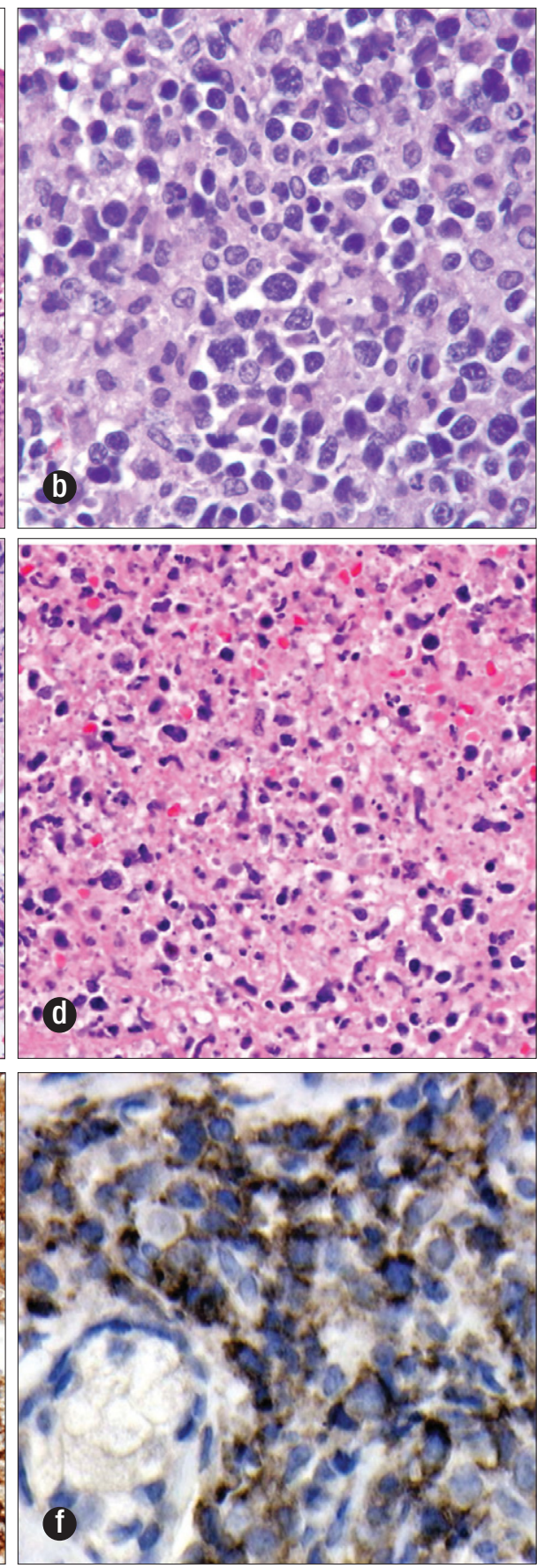

Figure 2. (a) Squamous mucosa with subjacent diffuse infiltrate by hematoxylin and eosin (H\&E) at $4 \times$. (b) Small- to medium-sized cells with irregular nuclei, inconspicuous nucleoli, and moderate clear cytoplasm by H\&E at $40 \times$. (c) Tumor cells within a blood vesse by H\&E at $40 x$. (d) Focal necrosis by H\&E at $40 x$. (e) CD3 at $10 \times$. (f) CD56 at 50×. (g) Epstein-Barr virus in situ hybridization at $40 x$ angiocentric T-cell lymphoma $(1,2)$. It is characterized by vascular damage and destruction, prominent necrosis, a cytotoxic phenotype, and an association with EBV. While most cases appear to be genuine NK-cell neoplasms, some cases have a cytotoxic T-cell phenotype (1).

Extranodal NK/T-cell lymphoma is rare in the United States and Europe. It is more prevalent in Asians, where it constitutes as much as $3 \%$ of non-Hodgkin lymphomas, and in Native Americans of Mexico, Central America, and South America (3). It occurs most often in adults, more commonly in men than women.

Little is known about the etiology. However, the strong association with EBV suggests a probable pathogenic role of the virus (1). The virus is present in a clonal episomal form with a type II latency pattern, similar to nasopharyngeal carcinoma and Hodgkin lymphoma, indicating that the tumor originates from a single EBV-infected cell $(3,4)$, and commonly shows a 30-base-pair deletion in the latent membrane protein-1 gene. How EBV gains entry is uncertain, since the tumor cells fail to express CD21, a surface protein that serves as the B-cell EBV receptor (3). Studies show that it is almost always of subtype $A$ and is present in $>95 \%$ of patients, irrespective of their ethnic origin (4). The disease activity can also be monitored by measuring circulating EBV DNA, as a high titer is correlated with extensive disease, unfavorable response to therapy, and poor survival (5). This lymphoma can also occur in the setting of immunosuppression, including transplant patients.

In terms of differential diagnosis, EBV studies can be very helpful. Nasal lymphomas of T-cell or B-cell origin have only a weak association with EBV, which further strengthens the argument for the etiological role of EBV in NK-cell tumors. Wegener granulomatosis is EBV negative. While lymphomatoid granulomatosis is EBV positive, it is a B-cell lymphoproliferative disease (4).

Clinically, patients present with symptoms of nasal obstruction or epistaxis due to a mass lesion, or extensive midfacial destruction. The lymphoma can extend 
to adjacent tissues including the orbit, as in this case. Bone marrow involvement is uncommon, though it may disseminate rapidly or be complicated by a hemophagocytic syndrome.

Histologically, mucosal sites often show extensive ulceration with a diffuse, permeative lymphomatous infiltrate and widely spaced or obliterated mucosal glands. An angiocentric and angiodestructive growth pattern is frequently present, with fibrinoid changes within blood vessels even in the absence of angioinvasion. Infarction-like coagulative necrosis and admixed apoptotic bodies are very common findings (2). The lymphoma can sometimes be accompanied by florid pseudoepitheliomatous hyperplasia of the overlying epithelium, mimicking well-differentiated squamous cell carcinoma (1).

Cytologically, the cells may be small, medium, large, or anaplastic. Most cases involve medium-sized cells or a mixture of small and large cells. The nuclei are often folded but can be elongated, with granular chromatin and a moderate amount of pale to clear cytoplasm. Mitotic figures are easily found, and Giemsa-stained touch preparations commonly highlight the azurophilic granules within the cytoplasm, which resemble those found in normal NK cells (3).

The typical immunophenotype is $\mathrm{CD} 2^{+}, \mathrm{CD} 56^{+}$, surface $\mathrm{CD}^{-}$, with cytoplasmic $\mathrm{CD} 3 \varepsilon^{+}$. Cytotoxic molecules (granzyme B, TIA-1, and perforin) are also positive. Other T- and NK-cellassociated antigens are usually negative, including CD4, CD5, CD8, TCR $\delta$, CD16, and CD57. EBER in situ hybridization demonstrates virtually all lymphoma cells as positive.

T-cell receptor and immunoglobulin genes are germline in most cases, and a clonal T-cell rearrangement may correspond to cytotoxic T lymphocyte derivation. Complex chromosomal abnormalities have been found in extranodal NK/T-cell lymphomas (1). The most common changes are $6 \mathrm{q}^{-}, 2 \mathrm{q}^{+}, 8 \mathrm{p}^{-}, 11 \mathrm{q}^{-}, 12 \mathrm{q}^{-}, 13 \mathrm{q}^{-}, 15 \mathrm{q}^{+}$, $17 \mathrm{q}^{+}$, and $22 \mathrm{q}^{+}$. No specific chromosomal translocation has been identified, although translocations involving the $8 \mathrm{p} 23$ breakpoint and $\operatorname{del}(6)(\mathrm{q} 21 ; \mathrm{q} 25)$ have been described $(1,6)$.

This lymphoma has a variable prognosis. A study by Li et al found that early stage disease tends to respond well to radiation therapy. However, the addition of chemotherapy does not significantly improve survival, and some patients die of disseminated disease despite aggressive therapy (7). Historically, the 5-year survival rate has been poor at $30 \%$ to $40 \%$, but it has recently improved to $71 \%$ with intensive therapy including up-front radiotherapy $(7,8)$. Significant unfavorable prognostic factors include advanced-stage disease (stage III or IV), unfavorable International Prognostic Index score, invasion of bone or skin, high-circulating EBV DNA levels, and the presence of EBV-positive cells in the bone marrow. The prognostic importance of cytological grade is unclear. The proliferative index, demonstrated by the Ki-67 immunostain, is usually high ( $>50 \%$ ), and studies suggest that it is associated with a bulky tumor burden and worse prognosis (9).

This patient presented with facial swelling and poor dental hygiene and, to further confound the situation, he had an undiagnosed alpha-thalassemia, the most likely cause of his splenomegaly. The biopsy of the infiltrating tumor proved to be CD3, CD56, TIA-1, granzyme B, and strong EBV positive with angioinvasion. It was also CD57 negative and consistent with the diagnosis of an extranodal NK/T-cell lymphoma, nasal type. It is unclear whether the CD3 positivity was cytoplasmic or surface antigen by immunohistochemistry, but CD3 epsilon cytoplasmic positivity is suggested by the negative T-cell gene rearrangement. The negative T-cell and heavy-chain gene rearrangements would be expected in extranodal NK/T cell lymphomas.

1. Chan J, Quintanilla-Martinez L, Ferry J, Peh SC. Extranodal NK/T-cell lymphoma, nasal type. In Swerdlow SH, Campo E, Harris NL, Jaffe ES, Pileri SA, Stein H, Thiele J, Vardiman JW, eds. WHO Classification of Tumours of Haematopoietic and Lymphoid Tissues. Lyon, France: IARC Press, 2008: 285-288.

2. Hutchison RE, Abraham NZ Jr. Leukocytic disorders. In McPherson RA, Pincus MR, eds. Henry's Clinical Diagnosis and Management by Laboratory Methods, 21st ed. Philadelphia: Saunders, 2007: 545-598.

3. Aster JC. Diseases of white blood cells, lymph nodes, spleen, and thymus. In Kumar V, Abbas AK, Fausto N, Aster J, eds. Robbins and Cotran Pathologic Basis of Disease, 8th ed. Philadelphia: Saunders, 2009.

4. Sun T. Natural killer cell lymphoma. In Flow Cytometry and Immunohistochemistry for Hematologic Neoplasms. Philadelphia: Lippincott Williams \& Wilkins, 2008: 267-277.

5. Lei KIK, Chan LYS, Chan WY, Johnson PJ, Lo YM. Quantitative analysis of circulating cell-free Epstein-Barr virus (EBV) DNA levels in patients with EBV-associated lymphoid malignancies. Br J Haematol 2000;111(1):239-246.

6. Wong N, Wong KF, Chan JK, Johnson PJ. Chromosomal translocations are common in natural killer-cell lymphoma/leukemia as shown by spectral karyotyping. Hum Pathol 2000;31(6):771-774.

7. Li YX, Yao B, Jin J, Wang WH, Liu YP, Song YW, Wang SL, Liu XF, Zhou LQ, He XH, Lu N, Yu ZH. Radiotherapy as primary treatment for stage IE and IIE nasal natural killer/T-cell lymphoma. J Clin Oncol 2006;24(1):181-189.

8. Cheung MM, Chan JK, Lau WH, Ngan RK, Foo WW. Early stage nasal NK/T-cell lymphoma: clinical outcome, prognostic factors, and the effect of treatment modality. Int J Radiat Oncol Biol Phys 2002;54(1):182190.

9. Kim SJ, Kim BS, Choi CW, Choi J, Kim I, Lee YH, Kim JS. Ki-67 expression is predictive of prognosis in patients with stage I/II extranodal NK/T-cell lymphoma, nasal type. Ann Oncol 2007;18(8):1382-1387. 